\title{
APRENDER METODOLOGIA QUALITATIVA EM PSICOLOGIA DA EDUCAÇÃO: PERCEÇÕES E EXPECTATIVAS DE ALUNOS DE MESTRADO
}

\author{
Ana P. Antunes ${ }^{1}$ e Sara B. Araújo ${ }^{2}$ \\ ${ }^{1}$ Universidade da Madeira, Centro de Investigação em Estudos da Criança Universidade do Minho, Portugal. \\ aantunes@uma.pt \\ ${ }^{2}$ Escola Superior de Educação Instituto Politécnico do Porto, Portugal. saraujo@ese.ipp.pt.
}

\begin{abstract}
Resumo. A metodologia qualitativa ainda costuma ser apresentada aos alunos como forma menos privilegiada de fazer investigação. Neste estudo pretendemos conhecer as perceções de alunos sobre a metodologia qualitativa e as suas expectativas sobre a Unidade Curricular de Métodos de Investigação II. Trata-se de um estudo qualitativo de natureza exploratória. Participaram 10 alunos (8 mulheres e 2 homens) de um curso de $2^{\circ}$ ciclo, em Psicologia da Educação, numa Universidade Pública Portuguesa, no ano letivo 2019/2020, tendo-se assegurado a participação informada e voluntária. Os dados foram recolhidos através de narrativas individuais, mediante orientações para a sua elaboração. Estas foram alvo de análise de conteúdo, por dois codificadores, numa lógica indutiva e dedutiva. Os dados revelaram algumas noções dos alunos sobre a metodologia qualitativa relacionadas, sobretudo, com o propósito da investigação, o processo metodológico, a natureza subjetiva da realidade, a perspetiva idiográfica e o papel de investigador participante. Além disso, as expectativas dos alunos sobre a Unidade Curricular eram positivas, destacando-se a possibilidade de aprendizagem de conhecimentos e competências e a aplicação prática dos conhecimentos, sobretudo, na dissertação de mestrado. Apesar de os alunos apresentarem algumas noções sobre a metodologia qualitativa, importa formação específica para aprofundamento e esclarecimento de alguns aspetos.
\end{abstract}

Palavras-chave: Investigação Qualitativa; Ensino-Aprendizagem; Psicologia da Educação.

\section{LEARNING QUALITATIVE RESEARCH IN EDUCATIONAL PSYCHOLOGY: PERCEPTIONS AND EXPECTATIONS OF MASTER'S DEGREE STUDENTS}

\begin{abstract}
Qualitative methodology is still presented to students as a less privileged way of doing research. In this study, we intend to examine the perceptions of students about the qualitative methodology and their expectations about the Curricular Unit of Research Methods II. This is a qualitative study of an exploratory nature. Participants were 10 students (8 women and 2 men) attending a 2nd cycle course, in Educational Psychology, at a Portuguese Public University, in the academic year 2019/2020. Informed and voluntary consent was ensured. The data were collected through individual narratives, following provided guidelines. Narratives were subject to content analysis, by two coders, in an inductive and deductive logic. Results revealed some notions of the students on the qualitative methodology that were mainly related with the purpose of the investigation, methodological process, subjective nature of the reality, idiographic perspective and the role of participating researcher. Moreover, students' expectations about the course were positive, highlighting the possibility of learning knowledge and skills, and the practical application of knowledge, especially in the master's dissertation. Although students present some notions about qualitative methodology, specific training is needed to deepen and clarify some aspects.
\end{abstract}

Keywords: Qualitative Research; Teaching-Learning; Educational Psychology.

\section{INTRODUÇÃO}

A visibilidade dos métodos de pesquisa qualitativa em Psicologia, nos Estados Unidos da América, aumentou de forma evidente com a disseminação da pesquisa qualitativa em periódicos e livros, com a constituição de organizações profissionais e científicas, e com o seu 
reconhecimento em instituições educacionais (Rubin, Bell, \& McClelland, 2018). Nesse sentido, a mais recente publicação do Manual de Publicação da American Psychological Association (2020), refere, pela primeira vez, padrões de escrita de pesquisas com recurso a métodos mistos e qualitativos.

No entanto, apesar desta divulgação, algumas questões se colocam sobre a formação de estudantes ao nível da graduação e pós-graduação (Rubin et al., 2018). De facto, a investigação quantitativa em relação à investigação qualitativa na área da Psicologia, tem ocupado um lugar predominante (Povee \& Roberts, 2014; Roberts \& Povee, 2014). Este facto tem, então, consequências ao nível do ensino. Por exemplo, o currículo de graduação em Psicologia, na maioria das universidades australianas, mantém um forte foco no ensino de métodos de pesquisa quantitativa (Roberts \& Castell, 2016).

Geralmente, os alunos de Psicologia contactam com a metodologia qualitativa após formação em metodologia quantitativa e assim tendem a desenvolver algumas crenças erradas sobre a investigação qualitativa (Povee \& Roberts, 2014) e a experimentar alguma dissonância cognitiva e maiores dificuldades em aprender métodos qualitativos (Roberts \& Castell, 2016). No ensino da metodologia qualitativa os professores debatem-se frequentemente com perguntas sobre "subjetividade" que invocam "preconceitos" dos recém-chegados à pesquisa qualitativa (Roulston \& Shelton, 2015).

O foco que privilegia o ensino da pesquisa qualitativa após a pesquisa quantitativa traduz uma perspetiva da pesquisa qualitativa como a metodologia "alternativa" (Roberts \& Castell, 2016).

Dada a complexidade dos métodos de pesquisa qualitativa, é possível referir que o ensino de abordagens qualitativas a estudantes de Psicologia apresenta desafios únicos. O ensino de métodos qualitativos, dada a ausência de uma linha única definida, pode confrontar-se com grandes divergências, de departamento para departamento, entre o modo como, e quais, os métodos qualitativos a ensinar (Gibson \& Sullivan, 2012). Além disso, o ensino da pesquisa qualitativa faculta oportunidades de aprendizagem experiencial e baseada na prática, sendo que, quando os alunos se envolvem nas experiências de aprendizagem, desenvolvem mudanças não só nas crenças sobre a pesquisa qualitativa, mas também transformações pessoais (Wagner, Kawulich, \& Garner, 2019). 
O envolvimento no processo de pesquisas qualitativas aumenta o desenvolvimento de muitas das habilidades psicoterapêuticas fundamentais nos alunos de Psicologia necessárias ao aconselhamento e terapia psicológica (Thorpe, 2013).

Neste trabalho, partindo da revisão da literatura e da lecionação de investigação qualitativa, pretende-se conhecer o que pensava um grupo de alunos do $1^{\circ}$ ano de mestrado de Psicologia da Educação sobre a metodologia qualitativa e o que esperava da Unidade Curricular (UC) de Métodos de Investigação com enfoque na metodologia qualitativa, antes do início da frequência da mesma.

\section{MÉTODO}

Este trabalho é de natureza exploratória e pretende-se realizá-lo em duas fases: antes do início da frequência da UC e no final da frequência da disciplina. Nesta primeira fase, objeto deste trabalho, procura-se responder a duas questões gerais: (1) que perceções têm os alunos sobre metodologia qualitativa; e (2) que expectativas têm sobre a disciplina de métodos de investigação qualitativa que vão frequentar.

\subsection{Participantes}

Neste trabalho participaram de forma voluntária 10 alunos (8 mulheres e 2 homens) de uma turma de 12 alunos da Unidade Curricular (UC) de Métodos de Investigação II (MI II), com idades compreendidas entre os 21 e os 30 anos. Os participantes frequentavam um curso de $2^{\circ}$ ciclo, em Psicologia da Educação, numa Universidade Pública Portuguesa, no ano letivo 2019/2020, estando a UC de MI II integrada no plano curricular do ciclo de estudos e contemplando uma matriz de investigação qualitativa.

\subsection{Materiais}

Narrativa: A opção para recolha de dados centrou-se na elaboração de uma narrativa por parte dos alunos, partindo a orientação de construção do texto das questões de investigação e da revisão da literatura, bem como de trabalho realizado anteriormente neste âmbito (Antunes, 2017). Assim, os alunos foram convidados a escrever uma narrativa onde abordassem os seguintes pontos:

1. Conhecimento sobre a metodologia qualitativa;

2. Comparação com a metodologia quantitativa; 
3. Importância da metodologia qualitativa;

4. Importância da metodologia qualitativa na Psicologia;

5. Expectativa sobre a Unidade Curricular;

6. Como penso que vai ser esta Unidade Curricular;

7. Importância da Unidade Curricular na formação do $2^{\circ}$ ciclo em Psicologia da Educação; e

8. Outro(s) aspeto(s) que queira referir.

\subsection{Procedimentos}

Na primeira aula de MI II, no início do semestre, antes da apresentação dos conteúdos programáticos e outra informação relacionada com a UC, foi solicitado aos alunos a realização de uma narrativa mediante a instrução referida no ponto anterior. Os alunos presentes na aula $(n=10)$, mediante consentimento informado, aceitaram participar e responder ao solicitado. Assim, a orientação para a realização da narrativa foi apresentada com recurso a vídeo projetor, numa folha escrita em Word, tendo sido distribuída por cada aluno uma folha em branco para a redação individual do texto, em conformidade com a instrução.

Posteriormente, as narrativas foram lidas na íntegra e alvo de análise de conteúdo na sequência sugerida por Bardin (2008): (1) pré-análise (organização e preparação do material) - os textos foram recolhidos, verificada a clareza da redação e codificados, atribuindo um código a cada participante; (2) exploração de materiais - as narrativas foram lidas e foi decidida a forma de análise das mesmas por acordo entre as investigadoras envolvidas na codificação; e (3) análise, inferência e interpretação dos resultados - os dados das narrativas foram analisados e discutidos à luz da literatura existente.

A análise de conteúdo decorreu, num primeiro momento, numa lógica dedutiva, tendo-se definido a priori sete categorias, a partir dos pontos organizadores da narrativa: (1) Conhecimento sobre a metodologia qualitativa; (2) Comparação com a metodologia quantitativa; (3) Importância da metodologia qualitativa; (4) Importância da metodologia qualitativa na Psicologia; (5) Expectativa sobre a Unidade Curricular; (6). Desenvolvimento da Unidade Curricular; (7) Importância da Unidade Curricular na formação do $2^{\circ}$ ciclo em Psicologia da Educação. 
A leitura dos textos permitiu perceber que os alunos responderam maioritariamente de forma sequencial aos tópicos, embora em alguns casos os estudantes tenham optado por agregar as respostas às questões explicitamente ou remetendo o leitor para uma resposta anterior.

Verificou-se ainda que em casos pontuais as narrativas eram omissas em relação a algum tópico e não emergiram conteúdos associados ao ponto 8, ou seja, Outro aspeto(s) que queira referir.

Assim, o processo de codificação decorreu, neste primeiro momento, com as investigadoras envolvidas, as autoras do estudo, a codificarem as narrativas individualmente, seguindo-se, um processo de discussão e comparação da categorização realizada.

Decorrente deste processo e por acordo entre as investigadoras, foi decidida uma nova análise dos dados, numa lógica dedutiva, agrupando alguns tópicos (1 e 2; e 5 e 6) dada a sobreposição, ou não distinção, verificada nas respostas dos alunos, acordando-se também a definição das categorias e subcategorias emergentes nas respostas dos alunos (baseada nas assunções filosóficas referidas por Creswell (2007) e na proposta de Merriam (1998), relacionadas com a metodologia qualitativa) para os seguintes tópicos:

(1) Caracterização da Metodologia Qualitativa;

(2) Importância da Metodologia Qualitativa;

(3) Importância da Metodologia Qualitativa na Psicologia;

(4) Expectativas sobre a Unidade Curricular; e

(5) Importância da UC no $2^{\circ}$ ciclo em Psicologia da Educação.

Assim, cada uma das investigadoras procedeu a nova categorização de forma individual, seguindo-se um processo de discussão e comparação das categorias e subcategorias até se alcançar acordo, também sobre a respetiva designação final (conforme tabelas na secção dos resultados).

$\mathrm{Na}$ apresentação dos dados, procurou-se assegurar o anonimato dos participantes, sendo que os excertos das narrativas aparecem assinaladas com a codificação atribuída, ou seja, a letra $\mathrm{A}$ (indicação de aluno) acompanhada de um número (numeração de 1 a 10 atribuída a cada participante). 


\section{RESULTADOS}

Os dados são apresentados a partir dos cinco tópicos definidos a priori e apresentados na secção anterior.

Os dados apresentados na Tabela 1 permitem verificar que os estudantes caracterizam a investigação qualitativa predominantemente pela referência ao seu propósito e a aspetos metodológicos.

Relativamente ao primeiro, ênfase é dada à geração de teorias, bem como à descrição e compreensão dos fenómenos.

Relativamente aos segundos, os participantes referiram de forma mais destacada aspetos relacionados com processos de recolha de dados e a lógica indutiva enquanto caracterizadora da $M Q$.

Tabela 1. Categorias e subcategorias para o tópico Caracterização da Metodologia Qualitativa.

\begin{tabular}{|c|c|c|}
\hline Categoria & Subcategoria (frequência) & Extratos das narrativas \\
\hline \multirow[t]{2}{*}{ Propósito da investigação } & $\begin{array}{l}\text { Gerar teorias }(A 1, A 2, A 4, A 5, \\
A 8, A 9, A 10)\end{array}$ & $\begin{array}{l}\text { "(...) trata-se de uma perspetiva que } \\
\text { procura desenvolver uma teoria } \\
\text { através da investigação de um } \\
\text { determinado fenómeno." (A1) }\end{array}$ \\
\hline & $\begin{array}{l}\text { Descrever/compreender (A6, } \\
\text { A8, A10) }\end{array}$ & $\begin{array}{l}\text { "(...) a metodologia qualitativa procura } \\
\text { descrever e compreender os } \\
\text { fenómenos." (A6) }\end{array}$ \\
\hline Dimensão do fenómeno & Idiográfica (A6, A7, A8, A9) & $\begin{array}{l}\text { "É um método de investigação que } \\
\text { visa o particular (...) a perceção } \\
\text { individual da situação em estudo." (A6) }\end{array}$ \\
\hline Pressuposto ontológico & Subjetividade (A1, A9) & $\begin{array}{l}\text { "(..) partindo, igualmente, da } \\
\text { perspetiva subjetiva de cada pessoa." } \\
\text { (A1) }\end{array}$ \\
\hline $\begin{array}{l}\text { Pressuposto } \\
\text { epistemológico }\end{array}$ & $\begin{array}{l}\text { Investigador participante (A2, } \\
A 3, A 9)\end{array}$ & $\begin{array}{l}\text { "A postura do investigador também } \\
\text { varia consoante a perspetiva } \\
\text { qualitativa e quantitativa, sendo que } \\
\text { na abordagem qualitativa o mesmo é } \\
\text { participante" (A2) }\end{array}$ \\
\hline \multirow[t]{3}{*}{ Pressuposto metodológico } & $\begin{array}{l}\text { Recolha de dados }(A 2, A 3, A 4, \\
A 7, A 8, A 9)\end{array}$ & $\begin{array}{l}\text { "(...) procurando recolher dados } \\
\text { através da observação direta dos } \\
\text { indivíduos, dos seus autorrelatos, } \\
\text { emoções, experiências, } \\
\text { comportamentos, atitudes e formas de } \\
\text { estar." (A3) }\end{array}$ \\
\hline & $\begin{array}{l}\text { Lógica indutiva ( } A 1, A 2, A 5, A 6 \text {, } \\
\text { A8) }\end{array}$ & $\begin{array}{l}\text { "Esta [MQ] explora os conceitos de } \\
\text { forma indutiva, e então no final ocorre } \\
\text { a elaboração de uma teoria." (A5) }\end{array}$ \\
\hline & $\begin{array}{l}\text { Processo/Procedimento } \\
\text { investigativo }(A 1, A 8, A 9)\end{array}$ & $\begin{array}{l}\text { (...) a qualitativa não formula } \\
\text { hipóteses, apenas em algumas } \\
\text { situações, e que serve como guias na } \\
\text { exploração." (A8) }\end{array}$ \\
\hline
\end{tabular}


No que concerne o tópico Importância da Metodologia Qualitativa, as narrativas destacam, novamente o propósito da investigação (sobretudo, a geração de teorias, e a descrição/ compreensão) bem como aspetos metodológicos, embora com menor frequência que no tópico anterior.

Tabela 2. Categorias e subcategorias para o tópico Importância da Metodologia Qualitativa.

\begin{tabular}{|c|c|c|}
\hline Categoria & Subcategoria (frequência) & Extratos das narrativas \\
\hline \multirow[t]{4}{*}{ Propósito da investigação } & Gerar teorias $(\mathrm{A} 1, \mathrm{~A} 2, \mathrm{~A} 5, \mathrm{~A} 8)$ & $\begin{array}{l}\text { "(...) sem este tipo de investigação } \\
\text { não seriam elaboradas as leis e } \\
\text { teorias que se mostram cruciais para } \\
\text { o mundo da investigação." (A5) }\end{array}$ \\
\hline & $\begin{array}{l}\text { Descrever/compreender (A4, } \\
A 6, A 9, A 10)\end{array}$ & $\begin{array}{l}\text { "Geralmente, pouco se conhece da } \\
\text { temática em questão, por isso faz-se } \\
\text { uma exploração do fenómeno, } \\
\text { descrevendo-o como ele se } \\
\text { comporta." (A6) }\end{array}$ \\
\hline & Avanço do conhecimento & "Esta metodologia é muito \\
\hline & científico (A2, $A 4, A 9)$ & $\begin{array}{l}\text { importante }(. . .) \text { contribuindo assim } \\
\text { para o avanço da ciência e do } \\
\text { conhecimento científico." (A2) }\end{array}$ \\
\hline Dimensão do fenómeno & Idiográfica (A8, A9) & $\begin{array}{l}\text { "A importância da metodologia } \\
\text { qualitativa reflete-se na forma como } \\
\text { esta estuda a experiência única de } \\
\text { cada sujeito" (A9) }\end{array}$ \\
\hline Pressuposto ontológico & Subjetividade (A1) & $\begin{array}{l}\text { "(...) permite estudar um } \\
\text { determinado fenómeno a partir da } \\
\text { subjetividade de cada pessoa (...)" }\end{array}$ \\
\hline \multirow[t]{3}{*}{$\begin{array}{l}\text { Pressuposto } \\
\text { metodológico }\end{array}$} & Recolha de dados (A3) & $\begin{array}{l}\text { "A metodologia qualitativa é muito } \\
\text { importante pois permite-nos obter } \\
\text { dados na primeira pessoa, dando- } \\
\text { nos assim a possibilidade de adquirir } \\
\text { conhecimento sobre as diferentes } \\
\text { perspetivas, comportamentos e } \\
\text { sentimentos de forma "pura", sem } \\
\text { manipulação." }\end{array}$ \\
\hline & Lógica indutiva (A8) & $\begin{array}{l}\text { "A metodologia qualitativa é de } \\
\text { grande importância pelo facto de } \\
\text { partir de um caso para o geral (...)" }\end{array}$ \\
\hline & $\begin{array}{l}\text { Processo/Procedimento } \\
\text { investigativo (A10) }\end{array}$ & $\begin{array}{l}\text { "(...) descrever fenómenos em } \\
\text { condições naturais, sem } \\
\text { necessidade de manipulação de } \\
\text { variáveis." }\end{array}$ \\
\hline
\end{tabular}

No que diz respeito à Importância da Metodologia Qualitativa na Psicologia, os resultados indicam menor frequência deste tópico nas narrativas analisadas. Os estudantes registaram, a este nível, a importância do propósito da investigação e do pressuposto metodológico.

A perspetiva idiográfica na metodologia qualitativa foi também referida por três participantes. 
Tabela 3. Categorias e subcategorias para o tópico Importância da Metodologia Qualitativa na Psicologia.

\begin{tabular}{|c|c|c|}
\hline Categoria & Subcategoria (frequência) & Extratos das narrativas \\
\hline \multirow[t]{2}{*}{ Propósito da investigação } & Gerar teorias $(\mathrm{A} 1, \mathrm{~A} 2, \mathrm{~A} 8)$ & $\begin{array}{l}\text { "Para a Psicologia, esta abordagem } \\
\text { tem um impacto significativo, uma } \\
\text { vez que permite formular novas } \\
\text { teorias (...)" (A1) }\end{array}$ \\
\hline & $\begin{array}{l}\text { Descrever/compreender (A4, } \\
\text { A6, A10) }\end{array}$ & $\begin{array}{l}\text { "(...) podemos compreender, } \\
\text { explicar e descrever fenómenos } \\
\text { psicológicos em contextos naturais } \\
\text { (...) contribuindo para um } \\
\text { conhecimento mais avançado." } \\
\text { (A10) }\end{array}$ \\
\hline Dimensão do fenómeno & Idiográfica (A6, A8, A9) & $\begin{array}{l}\text { “(...) esta metodologia permite } \\
\text { alcançar a perceção individual do } \\
\text { sujeito em relação a um determinado } \\
\text { assunto (...)" (A6) }\end{array}$ \\
\hline Pressuposto ontológico & Subjetividade (A1) & $\begin{array}{l}\text { "(...) tendo em conta a subjetividade } \\
\text { de cada pessoa." }\end{array}$ \\
\hline \multirow[t]{2}{*}{$\begin{array}{l}\text { Pressuposto } \\
\text { metodológico }\end{array}$} & Recolha de dados (A3, A7) & $\begin{array}{l}\text { "Este tipo de investigação é também } \\
\text { muito importante para a Psicologia, } \\
\text { uma vez que através das } \\
\text { observações diretas do investigador } \\
\text { é possível descodificar } \\
\text { comportamentos de diferentes } \\
\text { indivíduos, bem como as suas } \\
\text { experiências e emoções através dos } \\
\text { seus relatos." (A3) }\end{array}$ \\
\hline & $\begin{array}{l}\text { Processo/Procedimento } \\
\text { investigativo (A4) }\end{array}$ & $\begin{array}{l}\text { "É de realçar que esta abordagem } \\
\text { permite recolha de dados no } \\
\text { contexto em que ocorrem, ou seja, } \\
\text { não existe "interferências"." }\end{array}$ \\
\hline
\end{tabular}

No tópico Expectativas sobre a Unidade Curricular emergiram três categorias (Aspetos motivacionais, Processo de ensino-aprendizagem e Transferência da aprendizagem).

As subcategorias agregadas à categoria Processo de ensino-aprendizagem surgiram de forma destacada no discurso escrito, designadamente as expectativas positivas acerca da aprendizagem de conhecimentos e competências, bem como a menção a aspetos associados à prática pedagógica (como métodos de ensino e de avaliação). 
Tabela 4. Categorias e subcategorias para o tópico Expectativas sobre a Unidade Curricular.

\begin{tabular}{|c|c|c|}
\hline Categoria & Subcategoria (frequência) & Extrato de narrativa \\
\hline \multirow[t]{2}{*}{ Aspetos motivacionais } & $\begin{array}{l}\text { Predisposição emocional (A5, } \\
\text { A6, A8, A9) } \\
\text { Identificação com a MQ (A1, } \\
\text { A8, A9, A10) }\end{array}$ & $\begin{array}{l}\text { "Apesar de ainda não ter visto o } \\
\text { programa, é a disciplina que } \\
\text { aguardo com maior anseio (...)" (A6) } \\
\text { (...) acho que a minha "praia" é } \\
\text { mais a perspetiva qualitativa." (A10) }\end{array}$ \\
\hline & $\begin{array}{l}\text { Envolvimento nas atividades } \\
\text { pedagógicas ( } A 4, A 8, A 9)\end{array}$ & $\begin{array}{l}\text { "Penso que será uma UC trabalhosa } \\
\text { devido à natureza dos dados que } \\
\text { vamos trabalhar." (A4) }\end{array}$ \\
\hline $\begin{array}{l}\text { Processo de ensino- } \\
\text { aprendizagem }\end{array}$ & $\begin{array}{l}\text { Aprendizagem de } \\
\text { conhecimentos e } \\
\text { competências (A1, A2, A3, } \\
\text { A4, A5, A6, A7, A9) } \\
\text { Prática pedagógica (A3, A5, } \\
\text { A7, A8, A9, A10) }\end{array}$ & $\begin{array}{l}\text { “(..) criando em mim novas } \\
\text { competências que até hoje não tive } \\
\text { muita oportunidade de trabalhar } \\
\text { (...)" (A9) } \\
\text { "Penso que iremos trabalhar de que } \\
\text { forma se realiza uma investigação } \\
\text { qualitativa, ou seja, as etapas, os } \\
\text { processos, os recursos e a sua } \\
\text { importância. Penso também que } \\
\text { muito desse exercício será feito } \\
\text { através da realização de um } \\
\text { trabalho de investigação." (A5) }\end{array}$ \\
\hline \multirow[t]{3}{*}{$\begin{array}{l}\text { Transferência da } \\
\text { aprendizagem }\end{array}$} & $\begin{array}{l}\text { Aplicação na dissertação (A1, } \\
\text { A2, A3, A4) }\end{array}$ & $\begin{array}{l}\text { "(...) será uma mais valia para o } \\
\text { nosso conhecimento e para a } \\
\text { realização da nossa tese de } \\
\text { mestrado (...)" (A3) }\end{array}$ \\
\hline & $\begin{array}{l}\text { Aplicação na prática } \\
\text { profissional }(A 1, A 6)\end{array}$ & $\begin{array}{l}\text { "(...) e, acima de tudo, saber a } \\
\text { utilidade prática para a sua } \\
\text { implementação na vida de um } \\
\text { psicólogo." (A6) }\end{array}$ \\
\hline & $\begin{array}{l}\text { Aplicação noutros estudos } \\
\text { futuros (A4) }\end{array}$ & $\begin{array}{l}\text { "(...) de modo a poder utilizá-la, ou } \\
\text { não, (...) em trabalhos futuros." }\end{array}$ \\
\hline
\end{tabular}

A leitura da Tabela 5 permite verificar a perceção de importância ou mais valia atribuída à UC no $2 .^{\circ}$ ciclo de formação em Psicologia da Educação.

Os estudantes destacam, muito particularmente, as oportunidades para a transferência de aprendizagem quer a curto prazo (elaboração da dissertação de mestrado), quer a médio ou longo prazo (na prática profissional ou estudos futuros). 
Tabela 5. Categorias e subcategorias para o tópico Importância da UC no $2^{\circ}$ ciclo em Psicologia da Educação

\begin{tabular}{|c|c|c|}
\hline Categoria & Subcategoria (frequência) & Extrato de narrativa \\
\hline Aspetos valorativos & $\begin{array}{l}\text { Perceção de importância/mais } \\
\text { valia (A1, A2, A3, A4, A6, A7, A8, } \\
A 9, A 10)\end{array}$ & $\begin{array}{l}\text { "Esta unidade curricular será uma mais } \\
\text { valia para a descoberta do campo da } \\
\text { investigação (...)" (A10) }\end{array}$ \\
\hline $\begin{array}{l}\text { Processo de ensino- } \\
\text { aprendizagem }\end{array}$ & $\begin{array}{l}\text { Aprendizagem de conhecimentos } \\
\text { e competências (A1, A6, A7, A9) }\end{array}$ & $\begin{array}{l}\text { "Importante para obtermos } \\
\text { conhecimento de vários tipos de } \\
\text { metodologia de investigação que } \\
\text { existem e que podemos usar quando } \\
\text { formos fazer investigação, dá outra } \\
\text { perspetiva sobre como a investigação } \\
\text { pode ser feita." (A7) }\end{array}$ \\
\hline \multirow[t]{3}{*}{$\begin{array}{l}\text { Transferência da } \\
\text { aprendizagem }\end{array}$} & $\begin{array}{l}\text { Aplicação na dissertação (A1, A5, } \\
\text { A8, A10) }\end{array}$ & $\begin{array}{l}\text { "Nesta fase de preparação para } \\
\text { trabalharmos em breve a nossa tese de } \\
\text { mestrado, parece-me crucial termos } \\
\text { todas as noções necessárias acerca dos } \\
\text { tipos e processos de investigação." (A5) }\end{array}$ \\
\hline & $\begin{array}{l}\text { Aplicação na prática profissional } \\
\text { (A4, A6, A8, A9) }\end{array}$ & $\begin{array}{l}\text { "(..) para aprendermos técnicas de } \\
\text { investigação que nos possam auxiliar na } \\
\text { prática profissional." (A9) }\end{array}$ \\
\hline & $\begin{array}{l}\text { Aplicação noutros estudos } \\
\text { futuros }(A 2, A 10)\end{array}$ & $\begin{array}{l}\text { “(...) permitirá adquirir diversos } \\
\text { conhecimentos que iremos aplicar na } \\
\text { realização (...) [de] outros estudos.” (A2) }\end{array}$ \\
\hline
\end{tabular}

\section{DISCUSSÃO E CONCLUSÕES}

Os dados recolhidos permitem responder às questões de investigação, contribuindo para o alcance dos objetivos formulados, ou seja, conhecer as perceções dos alunos sobre a metodologia qualitativa, bem como as expectativas dos mesmos sobre a UC de métodos de investigação qualitativa, antes da sua frequência.

Mais concretamente, no que se refere às perceções dos alunos sobre a metodologia qualitativa encontraram-se dados relativos a três tópicos gerais, ou seja, a Caracterização da Metodologia Qualitativa, a Importância da Metodologia Qualitativa, e a Importância da Metodologia Qualitativa na Psicologia. Sobre o conhecimento que os alunos referem sobre a metodologia qualitativa verifica-se a ocorrência de aspetos relacionados com o Propósito da investigação, destacando-se o contributo na Geração de teorias e na Descrição e compreensão de fenómenos, com a Dimensão Idiográfica do fenómeno, bem como a perceção da natureza Subjetiva da realidade (Pressuposto ontológico) e o papel de Investigador participante (Pressuposto epistemológico).

Também o Pressuposto metodológico emerge na análise dos dados, caraterizado por especificidades na Recolha de dados e no Processo/procedimento investigativo, e adotante de uma Lógica indutiva, emergem. Estes alunos parecem revelar consciência da existência de especificidade e de diferenciação da metodologia qualitativa em comparação à 
metodologia quantitativa, referindo aspetos centrais da sua caracterização (Creswell, 2007; Merriam, 1998).

Na mesma linha apontam os dados recolhidos sobre a importância da metodologia qualitativa, ainda que aqui as narrativas sejam menos extensas e desenvolvidas, verificando-se, no geral, menos produções para estes tópicos. Contudo, importa destacar que não emergiu a referência ao Pressuposto epistemológico (Investigador participante), tendo-se verificado referências às outras quatro categorias encontradas para a Caracterização da metodologia qualitativa. De destacar que os alunos parecem reconhecer cientificidade à investigação qualitativa, quando falam sobre a sua importância, pois no Propósito da investigação emergiu o reconhecimento do seu contributo ao Avanço do conhecimento científico, sendo este um dado curioso, pois a literatura refere o condicionamento de alguns alunos sobre o rigor e validade da investigação qualitativa, quando iniciam a formação desta (Antunes, 2017; Povee \& Roberts, 2014; Roberts \& Castell, 2016).

No que se refere à importância da metodologia qualitativa na Psicologia as respostas dos alunos não parecem especificar e diferenciar sobremaneira o seu conteúdo do tópico anterior, pois as suas respostas são de alguma forma generalistas. A destacar, eventualmente, a referência à Dimensão do fenómeno em estudo numa perspetiva Idiográfica, ou seja, o reconhecimento da idiossincrasia e da singularidade do sujeito participante, aspeto importante o sujeito na ciência Psicológica. Tanto assim é que a própria Psicologia reconhece, atualmente, a importância desta metodologia (APA, 2020; Rubin et al., 2018). No entanto, em algumas narrativas parece estar patente uma escrita "livresca", queremos dizer, um relato baseado em conhecimento adquirido em manuais e não experienciado. Conhecimento esse que até, em alguns trechos, parece enviesado e dicotómico, como acontece em várias narrativas sobre a importância "exclusiva" da metodologia qualitativa para o desenvolvimento de teorias e para a exploração e abordagem inicial ao estudo de um fenómeno. Aqui, parecenos surgir de alguma forma o condicionamento da formação prévia, sobretudo, em investigação quantitativa, e também a necessidade de formação com enfâse na metodologia qualitativa e na especificidade da sua linguagem (Povee \& Roberts, 2014; Roberts \& Castel, 2016; Roberts \& Povee, 2014; Wagner et al., 2019).

Quanto à questão sobre as expectativas dos alunos sobre a UC de métodos de investigação qualitativa, antes da sua frequência, os dados foram agrupados em dois tópicos, isto é, 
Expectativas sobre a Unidade Curricular e Importância da UC no $2^{\circ}$ ciclo em Psicologia da Educação.

No que se refere às expectativas sobre a UC emergem três categorias diferenciadas mas interligadas e importantes no contexto da aprendizagem. Assim, nos Aspetos motivacionais, emerge a referência à Predisposição emocional dos alunos para frequentarem a UC, a Identificação com a $M Q$, ainda que não tenham conhecimento aprofundado da mesma, e o Envolvimento nas atividades pedagógicas, reconhecendo que será necessário trabalhar e corresponder às tarefas propostas. No Processo de ensino-aprendizagem, categoria mais referenciada pelos alunos, emergem aspetos sobre a Aprendizagem de conhecimentos e competências e sobre a Prática pedagógica, facto que nos parece compreensível, dado o contexto de aprendizagem em que se insere o objeto em estudo. E, na mesma lógica, aparece a Transferência da aprendizagem, destacando-se a Aplicação da aprendizagem efetuada na dissertação, na prática profissional ou noutros estudos futuros. Assim, este grupo de alunos parece ter a expectativa de aprender conhecimentos teóricos e práticos relacionados com a investigação qualitativa, esperando aplicá-los, sobretudo, na dissertação de mestrado, tarefa mais imediata que encontrarão e, quiçá, na atividade profissional e em estudos futuros. De referir ainda que parece um grupo motivado e empenhado em participar e envolver-se na UC.

Por último, a UC de métodos de investigação no $2^{\circ}$ ciclo em Psicologia da Educação é reconhecida como importante pelos alunos, através de três categorias que traduzem a sua perceção. Por um lado, encontram-se Aspetos valorativos, dando conta da Perceção de importância e/ou de mais valia da UC, por parte da esmagadora maioria dos alunos, ainda que nem sempre explicitem claramente porquê.

Por outro lado, encontra-se de alguma forma essa justificação quando se referem ao Processo de ensino-aprendizagem, destacando-se a Aprendizagem de conhecimentos e competências bem como a Transferência da aprendizagem, destacando-se, mais uma vez, a Aplicação da aprendizagem efetuada na dissertação, na prática profissional ou noutros estudos futuros, ainda que aqui não se destaque a aplicação na dissertação como no tópico anterior. Mais uma vez nos parece que estes dados traduzem a abertura dos alunos ao conhecimento e ao investimento nesta UC, antecipando-se que a frequência das aulas lhes possa fornecer conteúdos e competências de investigação para aprofundamento dos temas abordados, clarificando a possível aplicação ao mundo profissional (Thorpe, 2013; Wagner, et al., 2019). 
Apesar dos dados apresentados nos parecerem pertinentes não queremos deixar de referir algumas limitações do estudo. Por um lado, a instrução da narrativa pode permitir o seu questionamento ao apontar aspetos concretos orientadores, podendo contribuir para a convergência das respostas dos alunos.

No entanto, havia a oportunidade para a referência a respostas abertas pelos alunos, facto que não aconteceu, levando-nos a crer que não foi um fator condicionante, à semelhança do já verificado em Antunes (2017).

Por outro lado, sendo a primeira aula do semestre e não tendo os alunos familiaridade com a metodologia qualitativa, podem estes aspetos ter condicionado a elaboração das narrativas na medida em que a produção escrita poderia requerer e revelar domínio de conhecimentos bem como capacidades de síntese e de elaboração de texto.

Contudo, parece-nos que narrativas menos ricas e aprofundadas podem revelar sobretudo a novidade da Unidade curricular e, de facto, a sua necessidade na formação dos alunos.

Este trabalho é exploratório e constitui a primeira fase de um estudo que se quer continuado, ou seja, no final do semestre os alunos apreciarão a UC frequentada. Aí, esperamos ser possível comparar o desenvolvimento efetuado pelos alunos no que se refere a esta forma de fazer investigação, desmistificando e desenvolvendo conhecimentos e competências.

Agradecimentos. Este trabalho foi financiado por Fundos Nacionais através da FCT - Fundação para a Ciência e a Tecnologia no âmbito do projeto do CIEC (Centro de Investigação em Estudos da Criança da Universidade do Minho) com a referência UIDB/00317/2020.

\section{REFERÊNCIAS}

American Psychological Association (2020). Publication manual of the American Psychological Association. (7 $7^{\text {th }}$ ed). Washington, DC: Author. https://doi.org/10.1037/0000165-000.

Antunes, A. P. (2017). Formação académica em metodologia qualitativa: Prática pedagógica em Psicologia da Educação. Revista Lusófona de Educação, 36, 147-161. doi: 10.24140/issn.1645-7250.rle36.10.

Creswell, J. W. (2007). Qualitative inquiry and research design: Choosing among five approaches. (2 $2^{\text {nd }}$ ed). Thousand Oaks, CA: SAGE Publications.

Gibson, S., \& Sullivan, C. (2012). Teaching Qualitative Research Methods in Psychology: An introduction to the special issue. Psychology Learning and Teaching, 11(1), 1-5. http://dx.doi.org/10.2304/plat.2012.11.1.1.

Merriam, S. B. (1998). Qualitative research anda case study applications in education. San Francisco, CA: JosseyBass Publishers.

Povee, K., \& Roberts, L. D. (2014). Qualitative research in psychology: Attitudes of psychology students and 
academic staff. Australian Journal of Psychology, 66, 28-37. doi: 10.1111/ajpy.12031.

Roberts, L. D., \& Castell, E. (2016). "Having to Shift Everything We've Learned to the Side": Expanding Research Methods Taught in Psychology to Incorporate Qualitative Methods. Frontiers in Psychology, 7, 688. doi: 10.3389/fpsyg.2016.00688.

Roberts, L. D., \& Povee, K. (2014). A brief measure of attitudes towards qualitative research in psychology. Australian Journal of Psychology, 66, 249-256. doi: 10.1111/ajpy.12059.

Roulston, K., \& Shelton, S. A. (2015). Reconceptualizing Bias in Teaching Qualitative Research Methods. Qualitative Inquiry, 21(4), 332-342. https://doi.org/10.1177/1077800414563803.

Rubin, J. D., Bell, S., \& McClelland, S. I. (2018). Graduate education in qualitative methods in U.S. psychology: current trends and recommendations for the future. Qualitative Research In Psychology, 15(1), 29-50. https://doi.org/10.1080/14780887.2017.1392668 .

Thorpe, M. R. (2013). The process of conducting qualitative research as an adjunct to the development of therapeutic abilities in counselling psychology. New Zealand Journal of Psychology, 42(3), 35-43.

Wagner, C., Kawulich, B., \& Garner, M. (2019). A Mixed Research Synthesis of Literature on Teaching Qualitative Research Methods, 9(3), 1-18. https://doi.org/10.1177/2158244019861488. 